\title{
CardioMEMS: where we are and where can we go?
}

\author{
Issa Pour-Ghaz ${ }^{1}$, David Hana ${ }^{2}$, Joel Raja ${ }^{1}$, Uzoma N. Ibebuogu ${ }^{3}$, Rami N. Khouzam ${ }^{3}$ \\ ${ }^{1}$ Department of Internal Medicine, University of Tennessee Health Science Center, Memphis, TN, USA; ${ }^{2}$ Department of Internal Medicine, Kasr \\ Alainy School of Medicine, Cairo University, Cairo, Egypt; ${ }^{3}$ Division of Cardiovascular Diseases, Department of Internal Medicine, University of \\ Tennessee Health Science Center, Memphis, TN, USA \\ Contributions: (I) Conception and design: I Pour-Ghaz, RN Khouzam; (II) Administrative support: UN Ibebuogu, RN Khouzam; (III) Provision of \\ study materials or patients: None; (IV) Collection and assembly of data: I Pour-Ghaz, D Hana, J Raja; (V) Data analysis and interpretation: I Pour- \\ Ghaz, D Hana, J Raja; (VI) Manuscript writing: All authors; (VII) Final approval of manuscript: All authors. \\ Correspondence to: Issa Pour-Ghaz, MD. Internal Medicine Resident Physician, PGY-2, University of Tennessee Health Science Center, 956 Court \\ Ave., Suite H314, Memphis, TN 38163, USA. Email: ipourghaz@gmail.com.
}

\begin{abstract}
The prevalence of heart failure (HF) has been on the rise with associated increase in hospitalizations, morbidity and mortality. These hospitalizations have led to increasingly more cost on and decreased quality of life for patients. CardioMEMS is one of the newer devices designed to help tackle this issue by allowing for better monitoring of HF patients. This device also allows for accurate recording of pulmonary artery pressures (PAPs) and has also been applied in various other areas, such as aneurysmal tears, for monitoring pressures. In this manuscript we will review the current state of CardioMEMS HF system and investigate some of the other areas for its promising use in the field.
\end{abstract}

Keywords: CardioMEMS; pulmonary artery hypertension (PAH); congestive heart failure (CHF); heart failure reduced ejection fraction (HFrEF); heart failure preserved ejection fraction (HFpEF)

Submitted Jun 30, 2019. Accepted for publication Jul 15, 2019.

doi: $10.21037 / \mathrm{atm} .2019 .07 .53$

View this article at: http://dx.doi.org/10.21037/atm.2019.07.53

\section{Introduction}

Heart failure (HF) is a major health issue and a leading cause of hospitalization. The global prevalence has increased from 23 million in 2010 (1) to 40 million in 2015 (2). The aim of management of HF is to maintain the pumping function of the heart and prevent progression to decompensated $\mathrm{HF}$ and congestive sequelae which are the most common causes of hospitalization in HF patients (3). Throughout the past few decades the goal has been to find the best method for monitoring congestive symptoms. Fulfilling this task through regular physician visits, blood test and imaging modalities are insufficient for optimal control and decreasing the HF-related hospitalization rates (3). It has been shown, even markers such as $\mathrm{N}$-terminal pro-B type natriuretic (NT-proBNP) which is heavily relied on for diagnosis and guiding of management of $\mathrm{HF}$ and one that has been shown to have some value in mortality benefit (4), cannot reliably predict mortality or overall hospitalization rates (5). Thus, remote monitoring has emerged as a viable option to overcome the long interval between office visits to serve as a tool to provide an effective method to monitor patients remotely to 'catch' the disease in time in order to prevent hospitalization.

Non-invasive methods, such as consistent scheduled phone calls with the patients and electronic transfer of physiologic data through electronic devices, have been previously studied in order to monitor HF patients which have not shown significant impact on mortality or hospitalization rates $(6,7)$. Numerous clinical trials were conducted to see the impact of implantable cardiac electronic devices in the management of HF and early detection of congestive symptoms, however, the results were equivocal since the extraction of data was episodic or the extracted data, which were affected by variables such as heart rate, arrhythmia, changes in impedance, or patient activity, were not precise enough to allow early 
detection of fluid retention and timely management $(3,7)$. Acute decompensated heart failure (ADHF) pathophysiology gives rise to elevated ventricular filling pressure and has led to development of implantable hemodynamic monitoring devices that can monitor the right ventricular (RV) pressure and estimate the end diastolic pulmonary artery pressure (ePAD) which has a strong correlation with $\mathrm{HF}$ since it is the best representative of the left atrial pressure (LAP). Nevertheless, COMPASS-HF trial has showed nonsignificant difference compared to the optimal medical management (8). Nevertheless, this study has allowed us to understand the pathophysiology of both reduced and preserved ejection fraction HF more thoroughly (9). In both groups of HF, the ePAD was significantly elevated before the occurrence of ADHF (10). Therefore, continuous monitoring of the pulmonary artery pressure (PAP) could be used as an indicator of HF and would allow optimal monitoring and timely management before occurrence of symptomatic ADHF. CardioMEMS is a novel wireless device that was created for this purpose and approved by the Food and Drug Administration (FDA) in 2014 to help monitor PAP and send the data on daily basis to providers allowing timely management of HF to help lower the hospitalization rate (7).

\section{Application}

The CardioMEMS is a wireless pressure sensitive device that uses microelectromechanical systems (MEMS) technology. This device consists of an implantable HF sensor, a delivery catheter, and an electronic monitoring unit. Using right heart catheterization (RHC) and local anesthesia, the device is implanted in the distal pulmonary artery. It is composed of a coil and capacitor enveloped by a sealed silica capsule covered by silicone which form a circuit that resonates at a specific frequency. The changes in PAP applied on the sensor result in changes in the frequency of resonant waves. The device measures the pulmonary arterial pressure by ambient atmospheric pressure changes detected by its barometer. CardioMEMS does not have any batteries or leads and it is powered by an external antenna in the form of radiofrequency signals.

At home by using a portable electronic unit and a special pillow which contains an antenna, the information gathered is translated into pressure measurements and transmitted to a secure server for processing. The reading takes place when the antenna is held against the body or when the patient lies on the pillow. This process is pain free and does not result in any abnormal sensation during the reading. The electronic unit transmits the PA measurements daily. These data can be used by the physicians to adjust the HF therapy (mainly through adjusting the diuretic dose) before congestive symptoms develop which ultimately results in lower hospitalization rates (11). This device has shown significant reduction in hospitalization rates among patients with New York Heart Association (NYHA) Class III HF and is expected to show mortality benefit in the ongoing GUIDE-HF trial $(3,12,13)$. The current recommendation for its use is NYHA Class III HF patients who have been hospitalized for congestive heart failure (CHF) within one year prior to implantation.

CardioMEMS can detect pressure changes related to conditions other than CHF. A case of subclinical aortic infective endocarditis was diagnosed with the detection of early changes by the device (14). Additionally, the PA monitoring could be used to guide medical optimization of patients prior to left ventricle assist device (LVAD) implantation and help with early detection of complication $(15,16)$. Feldman and colleagues recently investigated the applicability of CardioMEMS as a tool for optimization of LVAD implantation timing and there was a shorter time LVAD implantation and it enables better optimization of LVAD performance when compared to current methods (17). Similar findings were reiterated by Tschöpe $e t a l$. in their case series whereby CardioMEMS led to better patient care when patients with end stage HF were transitioning to heart transplantation (18). In a 5-year cost effectiveness analysis of CardioMEMS, Schmier et al. established that it would be cost effective and it would lead to better quality adjusted life-year (QALY) for patient when compared to standard therapy (19). Other authors have also reported higher QALYs and cost-effectiveness when CardioMEMS is compared to standard therapy $(20,21)$. It is associated with very low device complications and initial studies have shown approximately a $1 \%$ complication rate related to the device and the most reported complication being bleeding from the puncture $(15,22)$. Overall complication rate during the first 3 years after FDA approval was approximately $2.8 \%$ with the most serious complications being PA injury and hemoptysis $(23,24)$. Singh and colleagues recently shown that despite its well established beneficial clinical outcomes for patients in management of HF, adaptation of CardioMEMS has been poor and among their patient population only $21 \%$ of patients eligible for device received it, pointing out that more 
education in this area is required (25).

\section{HF}

Hemodynamic monitoring guided therapy in HF can help decrease hospitalization and measuring PAP can be used to assess the volume status of the individual in HF. CardioMEMS is used for optimization of volume status with guided medical therapy in management of HF. CHAMPION trial was a prospective, parallel, single-blinded, multicenter study that enrolled participants with NYHA Class III HF symptoms with a history of admission to hospital due to HF. This study showed a significant reduction in hospitalizations due to HF exacerbations in group when monitoring of PAP was used to guide therapy (11). Rates of hospitalizations were reduced in the treatment group by $33 \%$ and there was a $47 \%$ lower mortality rate when compared with the control group (26). Furthermore, sub-group analysis of CHAMPION trial also revealed a decrease in HF admissions with PAP monitoring guided therapy compared to symptomatic therapy alone (27). Mortality benefit in addition to decrease in hospitalization was observed in cardiac resynchronization therapy patients (28). Furthermore, a retrospective analysis showed $48 \%$ reduction in HF exacerbation admissions in PAP monitoring guided therapy (29). In 2014, the US FDA granted approval for St. Jude to distribute CardioMEMS for implantable hemodynamic monitoring in NYHA class III heart failure preserved ejection fraction (HFpEF) and heart failure reduced ejection fraction (HFrEF) on optimal medical therapy.

An important variable for assessment off effectiveness of CardioMEMS in reduction of hospitalization was the 30day rate of readmission. Adamson and colleagues showed that in the study group, medications were changed more often as compared to the control group and the overall rate of hospitalizations was $49 \%$ lower in the treatment group when compared with control. It was also determined that all cause 30 -day readmissions were $58 \%$ lower in the treatment group (30). In a recent large observational study on 2000, researchers demonstrated that application of CardioMEMS led to greater reduction in PAP which could eventually lead to less hospitalizations in these patients due to the direct correlation between the two (31). Subset analysis of the CHAMPION trial has shown that the sensor can lead to better hemodynamic management of patients with preserved ejection fraction as well and its application led to lower rates of hospitalization when compared to standard therapy (32). Vanoli and colleagues argue that it is the even though symptomatic management of HF can help in some cases if caught early and prevent hospitalization, remote monitoring represents a key approach which can lead to very early detection of an issue. This is especially true because the level of burden is really high, especially in HF with preserved ejection fraction where effective therapies are missing (33). Another current trial investigating the effectiveness of CardioMEMS in long-term outcomes for NYHA Class III HF patients is the CardioMEMS Monitoring Study for Heart Failure, also known as the MEMS-HF trials. It is a prospective, non-randomized, open-label, multicenter study with primary endpoints of freedom from device complications and sensor failure at 12-month post-implantation, and secondary endpoints of annualized rate of HF-related hospitalizations at 12-month before and after implantation and assessment of QALY (34). It is the ability of CardioMEMS to be effective in catching cases early that makes it a great method for monitoring HF patients and such studies examining long-term outcomes in clinical settings help assess the effectiveness of this device in $\mathrm{HF}$ and long-term outcomes for patients.

\section{RHC vs. CardioMEMS}

The gold standard for diagnosis of pulmonary artery hypertension (PAH) and recording intracardiac pressures in HF patients is invasive hemodynamic assessment by RHC. Although it is an invasive procedure, it has become recognized and established as a safe method for assessment with minimal risks and has reported low morbidity and mortality in in patients with pulmonary hypertension (PH) (35). In an effort to minimize variations and errors in measurements, usually several readings are recorded at end-expiration while the patient is supine to allow for reproducibility, which is not an accurate assessment of patient's hemodynamics during their daily activities (36). Repeat hemodynamic assessment is often performed to assess disease progression and response to therapy which does put the patient at increased risk for complications such as infection and there are no definite recommendations regarding the timing for repeat assessments. The European Society of Cardiology guidelines for the treatment of PAH list serial RHCs as a Class IIa indication, Level of Evidence C (37). Data from a survey from $\mathrm{PH}$ specialists recommend repeat invasive hemodynamic measurements between 6 and 12 months after initiation of PAH-specific therapy and after changes in treatment (38). Though invasive, RHC is required for accurate diagnosis even though the readings 
obtained are not by any means representative of 'real-world' hemodynamics of the lungs. There are various noninvasive modalities for hemodynamic assessments such as brain natriuretic peptide testing, cardiac magnetic resonance imaging (MRI), and echocardiography, and CardioMEMS.

A meta-analysis in 2010 found six hemodynamic variables as markers of prognosis which include mean PA pressure, mean right atrial pressure, cardiac index, stroke volume index, peripheral vascular resistance, and mixed venous saturation (39). Although CardioMEMS was not designed to be specifically used as a management tool for PAH, it is the ongoing measurement of PA pressures that makes this device extremely valuable if the cardiac output could be derived from the sensor to calculate the total pulmonary resistance (40). It would allow for the same strategy used for titration of therapy applied for HF that could be applied to PAH treatment and guide PH therapy. Benza and colleagues performed a single center study investigating long-term pressure measurement and titration of therapy based on device interpretations showed that device guided therapy allowed for early recognition of optimal dosing when compared to the standard therapy and an enhanced cost saving due to lower drug dosing of up to $\$ 29,000$ and minimalization of prostacyclin-related side effects. They also showed that there was decreased risk due to lower need for RHC (41). Similarly, agreement between the HF sensor and RHC for systolic, diastolic, and mean PAPs has been shown to be excellent, with correlation coefficients of $0.94,0.85$, and 0.95 , respectively (all $\mathrm{P}<0.0001$ ) in a small study with 17 patients (38). A significant correlation of 0.90 was observed for the PA pressure measurement between CardioMEMS and Swan Ganz catheter (42). With CardioMEMS having the capability to accurately measure PA pressure as compared to RHC, it seems to be a safe, continuous alternative monitoring method in patients with $\mathrm{PAH}$ when compared to repeated invasive means of measurement of hemodynamic parameters with RHC, which can lead to higher rates of complications. In a subgroup analysis of the CHAMPION trial in patients with HF and World Health Organization group II PH showed that patients with $\mathrm{HF}$ without $\mathrm{PH}$ were at significantly lower risk for mortality than those with PH. In addition, PAP data knowledge and utilization in patients with and without PH lead to reduction in HF hospitalizations (43).

Noninvasive PA pressure monitoring by CardioMEMS can provide valuable data to physicians treating $\mathrm{PAH}$ which can vital when assessing response to therapy and modifying treatment. This can lead to earlier discovery of non-effective treatment therapies and overhaul of treatment if indicated or it can lead to lowest effective dose determination, which in turn translates into lower cost for the patient and lower likelihood side effects due to medication. By the same token, it also allows for lower number of necessary RHC and provision of a continuous cost-effective means of monitoring. The leading cause of mortality in PAH is eventual RV failure as a direct consequence of chronic pressure overload due to elevated lung pressures. One of the limitations of CardioMEMS device is the inability to measure right atrial and $\mathrm{RV}$ pressures as well as giving a good cardiac output estimate. This may limit its hemodynamic parameter assessment outside of PA pressure, but rising PA pressures should prompt titration or assessment of therapy. In addition, CardioMEMS can collect data continuously independent of the activity that the patient is undertaking as compared to the current gold standard which only obtains data in a resting state; thus, possibly provide more information regarding lung hemodynamics. In the future iterations of the device, the possibility of ability to record more hemodynamic variables cannot be ruled out and seems very likely. Thus, CardioMEMS can serve as a suitable indirect means of assessing pressure overload in patients with PAH.

\section{Conclusions}

Due to the ever-growing increasing costs associated with health care admissions, findings solutions for better monitoring of chronic conditions presents a very viable option. In the recent years there has been a series of new devices created for this reason and CardioMEMS is one of the therapeutic monitoring options. CardioMEMS has shown to be effective in prevention and reduction of HF hospitalizations in patients with HFrEF and HFpEF. It has been shown to be great option for hemodynamic monitoring in different locations such as aneurysms and atrial appendage. It is this versatility that makes CardioMEMS a great option for PAH monitoring to avoid the need for repeated cardiac catheterization. This application can also lead to easier monitoring of medication management in these patients and worth an investigation. With future iterations of this device and updates collection of more data points seems a very viable option and one that 
can be of great impact in the future.

\section{Acknowledgments}

None.

\section{Footnote}

Conflicts of Interest: The authors have no conflicts of interest to declare.

Ethical Statement: The authors are accountable for all aspects of the work in ensuring that questions related to the accuracy or integrity of any part of the work are appropriately investigated and resolved.

\section{References}

1. Bui AL, Horwich TB, Fonarow GC. Epidemiology and risk profile of heart failure. Nat Rev Cardiol 2011;8:30-41.

2. GBD 2015 Disease and Injury Incidence and Prevalence Collaborators. Global, regional, and national incidence, prevalence, and years lived with disability for 310 diseases and injuries, 1990-2015: a systematic analysis for the Global Burden of Disease Study 2015. Lancet 2016;388:1545-602.

3. Adamson PB, Abraham WT, Aaron M, et al. CHAMPION trial rationale and design: the long-term safety and clinical efficacy of a wireless pulmonary artery pressure monitoring system. J Card Fail 2011;17:3-10.

4. Lainchbury JG, Troughton RW, Strangman KM, et al. N-terminal pro-B-type natriuretic peptide-guided treatment for chronic heart failure: results from the BATTLESCARRED (NT-proBNP-Assisted Treatment To Lessen Serial Cardiac Readmissions and Death) trial. J Am Coll Cardiol 2009;55:53-60.

5. Tang WH, Girod JP, Lee MJ, et al. Plasma B-type natriuretic peptide levels in ambulatory patients with established chronic symptomatic systolic heart failure. Circulation 2003;108:2964-6.

6. Koehler F, Winkler S, Schieber M, et al. Impact of remote telemedical management on mortality and hospitalizations in ambulatory patients with chronic heart failure: the telemedical interventional monitoring in heart failure study. Circulation 2011;123:1873-80.

7. Abraham WT. Disease management: remote monitoring in heart failure patients with implantable defibrillators, resynchronization devices, and haemodynamic monitors.
Europace 2013;15 Suppl 1:i40-6.

8. Bourge RC, Abraham WT, Adamson PB, et al.

Randomized controlled trial of an implantable continuous hemodynamic monitor in patients with advanced heart failure: the COMPASS-HF study. J Am Coll Cardiol 2008;51:1073-9.

9. Mangi MA, Rehman H, Rafique M, et al. Ambulatory Heart Failure Monitoring: A Systemic Review. Cureus 2017;9:e1174.

10. Zile MR, Bennett TD, St John Sutton M, et al. Transition from chronic compensated to acute decompensated heart failure: pathophysiological insights obtained from continuous monitoring of intracardiac pressures. Circulation 2008;118:1433-41.

11. Givertz MM, Stevenson LW, Costanzo MR, et al. Pulmonary Artery Pressure-Guided Management of Patients With Heart Failure and Reduced Ejection Fraction. J Am Coll Cardiol 2017;70:1875-86.

12. Ayyadurai P, Alkhawam H, Saad M, et al. An update on the CardioMEMS pulmonary artery pressure sensor. Ther Adv Cardiovasc Dis 2019;13:1753944719826826.

13. St. Jude Medical Inc. CardioMEMS technology fact sheet. Available online: http://s1.q4cdn.com/166440495/files/ doc_downloads/Heart\%20Failure/Euope-CardioMEMSTechnology-Fact-Sheet.pdf

14. Sarsam S, Kaspar G, David S, et al. Early Detection of Subclinical Aortic Valve Endocarditis with the CardioMEMS Heart Failure System. Am J Case Rep 2017;18:665-8.

15. Veenis JF, Birim O, Brugts JJ. Pulmonary artery pressure telemonitoring by CardioMEMS in a patient pre- and post-left ventricular assist device implantation. Eur J Cardiothorac Surg 2019. [Epub ahead of print].

16. Veenis JF, Manintveld OC, Constantinescu AA, et al. Design and rationale of haemodynamic guidance with CardioMEMS in patients with a left ventricular assist device: the HEMO-VAD pilot study. ESC Heart Fail 2019;6:194-201.

17. Feldman DS, Moazami N, Adamson PB, et al. The Utility of a Wireless Implantable Hemodynamic Monitoring System in Patients Requiring Mechanical Circulatory Support. ASAIO J 2018;64:301-8.

18. Tschöpe C, Alogna A, Spillmann F, et al. The CardioMEMS system in the clinical management of endstage heart failure patients: three case reports. BMC Cardiovasc Disord 2018;18:155.

19. Schmier JK, Ong KL, Fonarow GC. Cost-Effectiveness of Remote Cardiac Monitoring With the CardioMEMS 
Heart Failure System. Clin Cardiol 2017;40:430-6.

20. Sandhu AT, Goldhaber-Fiebert JD, Owens DK, Turakhia MP, Kaiser DW, Heidenreich PA. Cost-Effectiveness of Implantable Pulmonary Artery Pressure Monitoring in Chronic Heart Failure. JACC Heart Fail 2016;4:368-75.

21. Cowie MR, Simon M, Klein L, Thokala P. The costeffectiveness of real-time pulmonary artery pressure monitoring in heart failure patients: a European perspective. Eur J Heart Fail 2017;19:661-9.

22. Abraham WT, Stevenson LW, Bourge RC, et al. Sustained efficacy of pulmonary artery pressure to guide adjustment of chronic heart failure therapy: complete follow-up results from the CHAMPION randomised trial. Lancet 2016;387:453-61

23. Vaduganathan M, DeFilippis EM, Fonarow GC, et al. Postmarketing Adverse Events Related to the CardioMEMS HF System. JAMA Cardiol 2017;2:1277-9.

24. Rali AS, Shah Z, Sauer AJ, et al. Hemoptysis After CardioMEMS Implantation: Case Report and Review. Am J Case Rep 2018;19:382-5.

25. Singh R, Varjabedian L, Kaspar G, et al. CardioMEMS in a Busy Cardiology Practice: Less than Optimal Implementation of a Valuable Tool to Reduce Heart Failure Readmissions. Cardiol Res Pract 2018;2018:4918757.

26. Abraham WT, Adamson PB, Bourge RC, et al. Wireless pulmonary artery haemodynamic monitoring in chronic heart failure: a randomised controlled trial. Lancet 2011;377:658-66.

27. Goldberg LR, Desai AS, Costanzo MR, et al. Pressure for action: implantable pulmonary artery pressure sensor measurements alone beat clinical signs to guide prevention of heart failure hospitalizations. Heart Rhythm 2015;12:S82-3.

28. Adamson PB, Abraham W, Stevenson L, et al. Benefits of pulmonary artery pressure monitoring extend to reduction of all-cause rehospitalization. J Am Coll Cardiol 2014;63:A746.

29. Desai AS, Bhimaraj A, Bharmi R, et al. Reduction in Heart Failure Hospitalizations With Ambulatory Hemodynamic Monitoring Seen in Clinical Trials Is Maintained in the 'Real World'. J Am Coll Cardiol 2017. doi: 10.1016/ j.jacc.2017.03.009.

30. Adamson PB, Abraham WT, Stevenson LW, et al. Pulmonary Artery Pressure-Guided Heart Failure Management Reduces 30-Day Readmissions. Circ Heart Fail 2016. doi: 10.1161/ CIRCHEARTFAILURE.115.002600.
31. Heywood JT, Jermyn R, Shavelle D, et al. Impact of Practice-Based Management of Pulmonary Artery Pressures in 2000 Patients Implanted With the CardioMEMS Sensor. Circulation 2017;135:1509-17.

32. Adamson PB, Abraham WT, Bourge RC, et al. Wireless pulmonary artery pressure monitoring guides management to reduce decompensation in heart failure with preserved ejection fraction. Circ Heart Fail 2014;7:935-44.

33. Vanoli E, D'Elia E, La Rovere MT, et al. Remote heart function monitoring: role of the CardioMEMS HF System. J Cardiovasc Med (Hagerstown) 2016;17:518-23.

34. Angermann CE, Assmus B, Anker SD, et al. Safety and feasibility of pulmonary artery pressure-guided heart failure therapy: rationale and design of the prospective CardioMEMS Monitoring Study for Heart Failure (MEMS-HF). Clin Res Cardiol 2018;107:991-1002.

35. Hoeper MM, Lee SH, Voswinckel R, et al. Complications of right heart catheterization procedures in patients with pulmonary hypertension in experienced centers. J Am Coll Cardiol 2006;48:2546-52.

36. Kovacs G, Avian A, Pienn M, et al. Reading pulmonary vascular pressure tracings. How to handle the problems of zero leveling and respiratory swings. Am J Respir Crit Care Med 2014;190:252-7.

37. Galiè N, Humbert M, Vachiery JL, et al. 2015 ESC/ERS Guidelines for the diagnosis and treatment of pulmonary hypertension: The Joint Task Force for the Diagnosis and Treatment of Pulmonary Hypertension of the European Society of Cardiology (ESC) and the European Respiratory Society (ERS): Endorsed by: Association for European Paediatric and Congenital Cardiology (AEPC), International Society for Heart and Lung Transplantation (ISHLT). Eur Heart J 2016;37:67-119.

38. Abraham WT, Adamson PB, Hasan A, et al. Safety and accuracy of a wireless pulmonary artery pressure monitoring system in patients with heart failure. Am Heart J 2011;161:558-66.

39. Swiston JR, Johnson SR, Granton JT. Factors that prognosticate mortality in idiopathic pulmonary arterial hypertension: a systematic review of the literature. Respir Med 2010;104:1588-607.

40. Davey R, Raina A. Hemodynamic monitoring in heart failure and pulmonary hypertension: From analog tracings to the digital age. World J Transplant 2016;6:542-7.

41. Benza RL, Doyle M, Cham M, et al. A Study to Explore the Feasibility and Safety of Using an Implantable Hemodynamc Monitor in PAH Patients. J Heart Lung Transplant 2015;34:S142. 
42. Verdejo HE, Castro PF, Concepcion R, et al. Comparison of a radiofrequency-based wireless pressure sensor to swan-ganz catheter and echocardiography for ambulatory assessment of pulmonary artery pressure in heart failure. J Am Coll Cardiol 2007;50:2375-82.

Cite this article as: Pour-Ghaz I, Hana D, Raja J, Ibebuogu UN, Khouzam RN. CardioMEMS: where we are and where can we go? Ann Transl Med 2019;7(17):418. doi: 10.21037/ atm.2019.07.53
43. Benza RL, Raina A, Abraham WT, et al. Pulmonary hypertension related to left heart disease: insight from a wireless implantable hemodynamic monitor. J Heart Lung Transplant 2015;34:329-37. 